\title{
In Vitro Evaluation of Drug Release from Modified Release Delivery Systems: Initial Experiences with Calibrators for USP Dissolution Apparatus 3.

\section{Abstract}

USP 23 now includes two new apparatus (Apparatus 3 and 4) designed for the purpose of characterizing drug release from extended-release dosage forms. These new apparatus permit changes in media during the evaluation and expose the delivery system to different bydrodynamics. Apparatus 3, which is the focus of this experimental work, consists of 7 reciprocating cylinders that can be moved through multiple (6) rows of cylindrical vessels; dip rate, time of immersion in each row, and the specific media employed are primary factors which can be manipulated. The aim of this study was to compare two calibrators [e.g., USP Chlorpheniramine Extended-release Tablets (Drug Release Calibrator, single unit) and USP Theophylline Extended-release Beads (Drug Release Calibrator, multiple unit)] in Apparatus 3 (VanKel BIO-DIS) using single and multiple row procedures. Despite the multiple row design, the Apparatus Suitability Test for both calibrators is conducted by sampling from vessels in a single row. This report demonstrates that results for both calibrators fall within specified limits when multiple rows of vessels are used.

\section{Background}

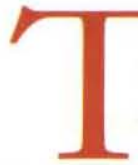

he United States Pharmacopeia (USP) has recently included a new dissolution apparatus (Apparatus 3) for drug release testing as an alternative method to the now classical basket and paddle apparatus. USP conducted a collaborative study to establish the system suitability limits for two new calibrators that are now available for use: USP Chlorpheniramine Extended-release Tablets (Drug Release Calibrator, single unit) and USP Theophylline Extendedrelease Beads (Drug Release Calibrator, multiple unit. The USP protocol sheet for each calibrator includes acceptance ranges which were reviewed and approved by the USP Subcommittee on Dissolution, Bioavailability, and Bioequivalence, and subsequently by the USP Reference Standards Committee. Calibration normally encompasses a complete check for mechanical properties of the apparatus, performance of the USP Apparatus System Suitability Test, and interpretation of the results.

Apparatus 3 offers the analyst numerous options: altering agitation conditions (e.g., selection of different mesh screens for the sample cell and setting the dipping rate) for the run; periodic exposure to new and/or different media (e.g., moving the sample cells to a new "row" of reservoir vessels); variable exposure time for each row of reservoir vessels and for the entire duration of testing; and sampling from the vessels during the programmed intervals if desired.

The flexibility of the apparatus is a positive attribute for pharmaceutical scientists engaged in the quality assessment of controlled release delivery systems and/or the development of in vitro - in vivo correlations which might serve as surrogates for bioequivalence testing. However, with increased flexibility comes increased complexity making calibration and validation of the apparatus a challenge.

Despite the fact that the apparatus has a multiple row design, the Apparatus Suitability Test for both calibrators is conducted by sampling from vessels in a single row. The aim of this brief study was to compare two calibrators [e.g., USP 


\section{A Recommendation for Reduced Testing....cont.}

Chlorpheniramine Extended-release Tablets (Drug Release Calibrator, single unit) and USP Theophylline Extended-release Beads (Drug Release Calibrator, multiple unit)] in Apparatus 3 using single and multiple row procedures.

\section{Experimental}

Chlorpheniramine Extended-release Tablets (Drug Release Calibrator, single unit) and USP Theophylline Extended-release Beads (Drug Release Calibrator, multiple unit) were analyzed using USP Apparatus 3 (VanKel BIO-DIS), under the conditions summarized in Table 1.

Apparatus 3 (VanKel BIO-DIS Extended Release Tester) was set up according to the manufacturers specifications. The sample cells were assembled as specified with a 20 mesh, 0.84 $\mathrm{mm}$ stainless steel screen on the bottom of each of the reciprocating cylinders and no screen on top. After the test sample (beads or tablet) was weighed and placed in the dry cell, the top of the cylinder was secured and then the assembly was attached to the appropriate reciprocating rod.

The dissolution medium in these tests was warmed to $37^{\circ} \mathrm{C}$ and degassed (stirring under vacuum) for at least 10 minutes prior to instrument set-up. To keep the temperature of the medium in the vessels at $37^{\circ} \mathrm{C}$ the water bath temperature controller was set to $37.5^{\circ} \mathrm{C}$. An initial volume of $250 \mathrm{~mL}$ of the warm degassed medium was placed in each cylindrical vessel, and the vessel was then placed in the apparatus. At the completion of this operation, the retractable plastic cover was secured in place covering all the vessels except those in the active row.

\section{Table 1. Experimental conditions for each calibrator.}

\begin{tabular}{lll} 
& \multicolumn{1}{c}{$\begin{array}{c}\text { Theophylline Extended- } \\
\text { release Beads }\end{array}$} & \multicolumn{1}{c}{$\begin{array}{c}\text { Chlorpheniramine } \\
\text { Extended-release Tablets }\end{array}$} \\
\hline Apparatus & VanKel BIO-DIS Extended Release Tester & VanKel BIO-DIS Extended Release Tester \\
Dissolution Medium & $0.1 \mathrm{~N} \mathrm{HCl}$ at $37^{\circ} \mathrm{C}$ & Water at $37^{\circ} \mathrm{C}$ \\
Volume & $250 \mathrm{~mL}$ & $250 \mathrm{~mL}$ \\
Speed & $15 \mathrm{DPM}$ & 5 or $30 \mathrm{DPM}$ \\
Sampling Time & $2+4 \mathrm{hours}$ & $1+3$ hours or $2+4$ hours \\
Sampling & $10 \mathrm{ml}$ aliquot & $10 \mathrm{ml}$ aliquot $+60 \mathrm{\mu L} \mathbf{0 . 1 \mathrm { N } \mathrm { HCl }}$ \\
Analysis & UV Spectrophotometry (Perkin - Elmer, Lambda - 3B) \\
\hline
\end{tabular}

Table 2. USP Specified Limits for the calibrators.

\begin{tabular}{|c|c|c|}
\hline \multicolumn{3}{|c|}{$\begin{array}{l}\text { USP DRUG RELEASE CALIBRATOR, } \\
\text { MULTIPLE-UNIT TYPE (APPARATUS 3) } \\
\text { Theophylline Extended-release Beads, Lot F-1 }\end{array}$} \\
\hline \multicolumn{2}{|c|}{ Time in hours } & $\begin{array}{l}\text { \% Dissolved } \\
15 \text { Dips per Minute }\end{array}$ \\
\hline & $16-25$ \\
\hline six & & $61-92$ \\
\hline \multicolumn{3}{|c|}{$\begin{array}{l}\text { USP DRUG RELEASE CALIBRATOR, } \\
\text { SINGLE UNIT TYPE (APPARATUS 3) }\end{array}$} \\
\hline Time in hours & $\begin{array}{l}\text { \% Dissolved } \\
5 \text { Dips per Minute }\end{array}$ & $\begin{array}{l}\text { \% Dissolved } \\
30 \text { Dips per Minute }\end{array}$ \\
\hline one & $22-30$ & - \\
\hline two & - & $37-61$ \\
\hline four & 51-67 & - \\
\hline six & - & NLT 79 \\
\hline
\end{tabular}




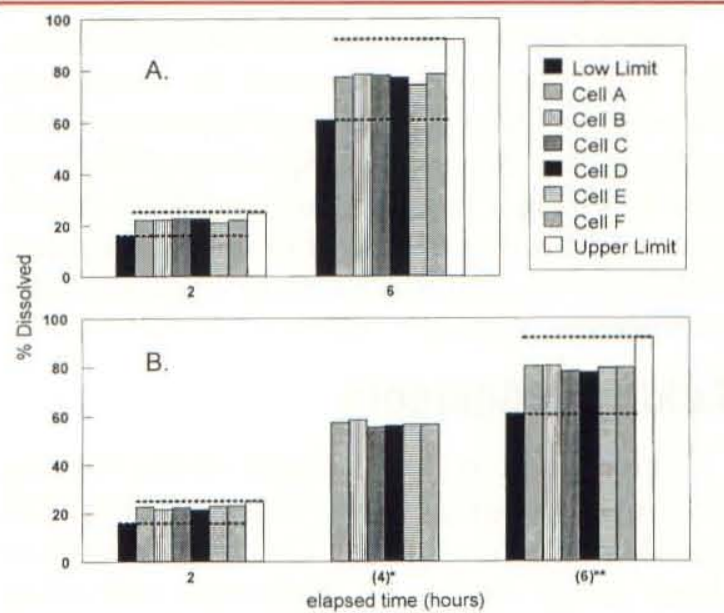

Figure 1. Theopbylline Extended-release Beads in Apparatus 3 at 5 dips per minute. A. Single Row with sampling; B. Two rows.

* Percent dissolved in the second row for a 4 bour period.

** Cumulative percent dissolved based on a summation of results from rows 1 and 2 .

\section{Results}

Figures 1-3 summarize the results of this experimental work. Each of these figures represents results of experiments conducted according to the Apparatus Suitability Test (Graph

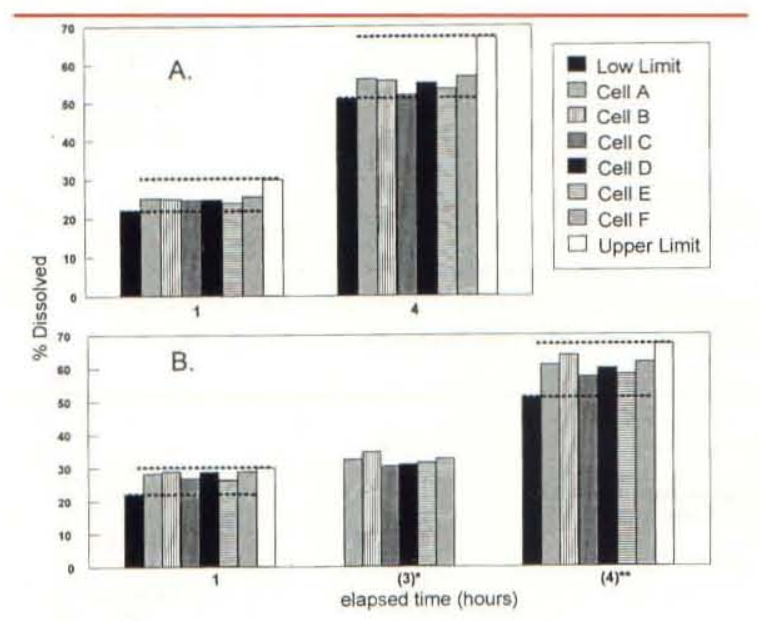

Figure 2. Chlorpheniramine Maleate Extended- release Tablets in Apparatus 3 at 5 dips per minute. A. Single Row with sampling; $B$. Two rowvs.

* Percent dissolved in the second row for a 4 bour period. ** Cumulative percent dissolved based on a summation of results from rows 1 and 2 .

A), where only a single row of the apparatus is utilized. Also included are results for the tests conducted by utilizing two rows of vessels (Graph B), with exposure times guided by the test criteria. 


\section{In Vitro Evaluation of Drug Release....cont.}

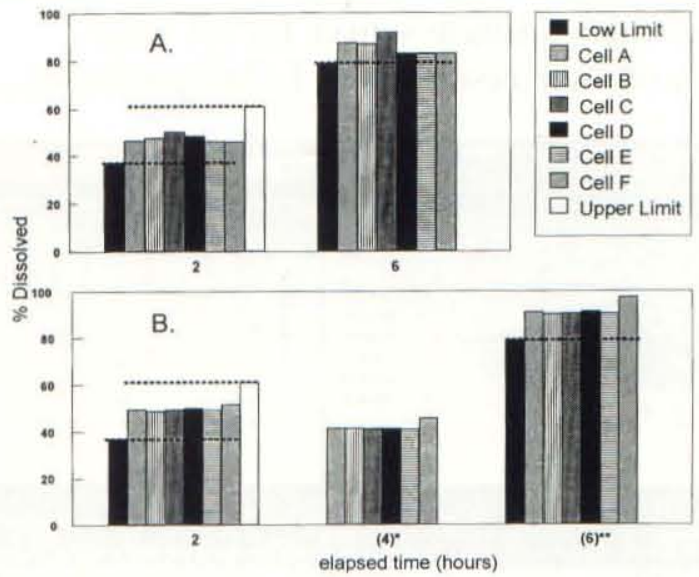

Figure 3. Chlorpheniramine Maleate Extended-release Tablets in Apparatus 3 at 30 dips per minute. A. Single Row with sampling; $B$. Two rows.

* Percent dissolved in the second row for a 4 bour period.

** Cumulative percent dissolved based on a summation of results from rows 1 and 2 .
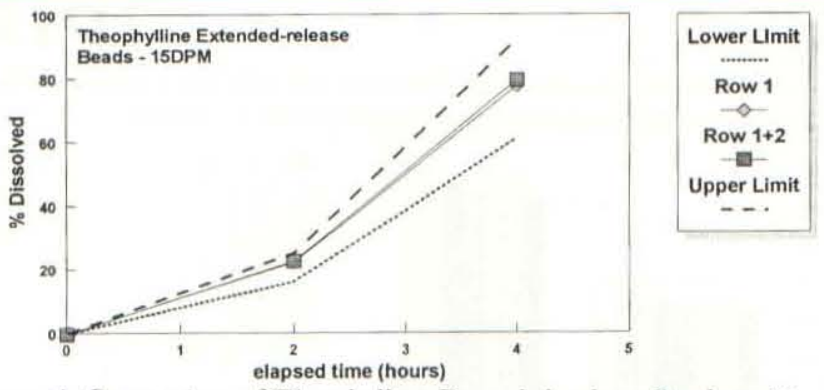

Figure 4. Comparison of Theophylline Extended-release Beads at 15 DPM using single and multiple row methods.
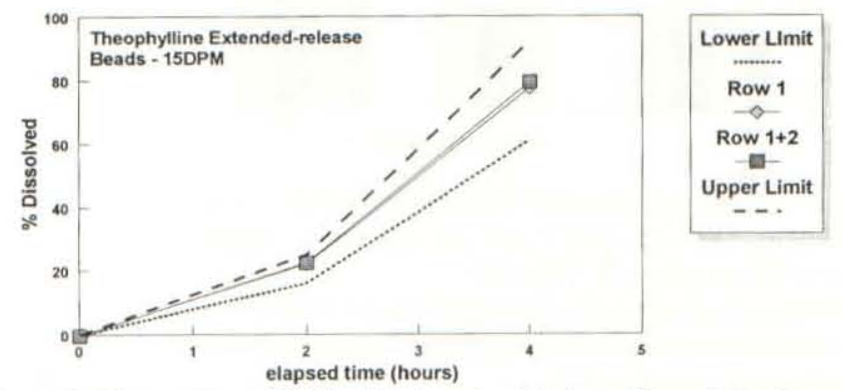

Figure 5. Comparison of Chlorpheniramine Maleate Extended-release Tablets at 5 and 30 DPM using single and multiple row methods.

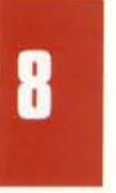

Figures 4-5 also summarize the data, in this case representing the release results for the Theophylline beads and Chlorpheniramine Maleate tablets as average values for the six replicates plotted against time, and including the limits of the Apparatus Suitability Test.

\section{Conclusions}

Data from single and multiple row experiments in USP Dissolution Apparatus 3 demonstrate that both calibrators fall within specified USP limits. However, for all calibrators under all conditions the $\%$ dissolved at a particular time point is greater when the experiment is conducted by moving the sample chambers to a new row. This result is consistent with the establishment of sink conditions (e.g., no dissolved drug) when the samples are moved to the second row, Also, results calculated from the single row test did not show as much cell to cell variation as compared to the results from test runs using two rows. At 30 DPM, a loss of approximately $15-18 \mathrm{mls}$ of dissolution media, apparently through the air holes, was observed. In some instances, calibrator tablets were found to be adhering to the screen at 5 DPM using the single row.

These conclusions are based on limited data, and more testing is required to completely characterize the performance of Apparatus 3. It is unlikely that the current Apparatus Suitability Test is sufficient in this regard, and USP should give consideration to designing and conducting a collaborative, multi-lab study that includes multiple row testing.

\section{Acknowledgments}

This work was done as part of the Visiting Scientist program in the UMAB/FDA Collaborative Agreement. The authors wish to thank James Leslie, Ed Krikorian, and other members of the University of Maryland at Baltimore Pharmacokinetics Biopharmaceutics Laboratory (PBL) for their assistance in conducting this project. The authors also wish to thank Brian Rohrs, Ph.D, of Pharmacia \& Upjohn, Kalamazoo, Michigan for his comments on this project.

\section{References}

1. The United States Pharmacopeia, 23rd Edition, Chapter <711>, <724>, USPC, Rockville, 1995. 